\title{
Biomasa y producción de Mixodiaptomus laciniatus (Copepoda, calanoida) en el lago de La Caldera (Sierra Nevada, Granada)
}

\author{
Luis Cruz-Pizarro
}

Departamento de Ecología. Facultad de Ciencias. Universidad de Granada.

\author{
SUMMARY \\ BIOMASS AND PRODUCTION OF Mixodiaptomus lacintatus (COPEPODA, CALANOIDA) \\ IN THE LAKE "LA CALDERA" (SIERRA NEVADA, SE SPAIN).
}

$M$. laciniatus is the dominant species in the zooplankton community of the lake La Caldera. Its life cycle was studied and its biomass and production estimated from periodical samples collected in the pelagic zone of the lake during the ice-free period in 1977. This population behaves in La Caldera as acyclic and univoltine. The duration of the main generation was estimated in about 52-56 days and mean duration of instars was included between 0.8 (N1) and more than 15 days (CV). The decrease in population size is described by the equation:

$$
\mathrm{N}_{\mathrm{t}}=14554.6 \cdot \mathrm{e}^{-0.00528 \mathrm{t}}
$$

The daily mortality rate was $m=0.528 \%$, close to the value obtained by REY \& CAPBLANCQ (1975) for this species in a similar ecosystem.

Biomass increases continuously during the existence period of the population, with values ranging from $8.13 \cdot 10^{-4}$ to $67.66 \mathrm{mg} / \mathrm{m}^{3}$. Production also increases with time, until the maximum population density is reached. At this moment; $P=3.18 \mathrm{mg} / \mathrm{m}^{3}$. day. From then on, it decreases sligthtly and is maintained at relatively high values, until the end of the sampling period. The rate Production/Biomass ( $\left.\mathrm{P}^{\prime} / \mathrm{B}\right)$ decreases as the population develops, reaching the maximum (1.60) at the begnning of the sampling.

\section{INTRODUCCION}

La Caldera es un lago situado en la zona de la tundra de Sierra Nevada, a $3.050 \mathrm{~m}$. de altitud, que presenta características propias de un ecosistema acuático oligotrófico alpino: aguas frías poco mineralizadas (en las que el fosfato parece ser el principal factor limitante para el desarrollo de las algas) y bajos valores de productividad primaria (inferiores a $0,2 \mathrm{mg} \mathrm{C} / \mathrm{m}^{3}$ hora). Durante un período de 8 a 10 meses permanece cubierto de hielo, con un corto periodo de condiciones favorables para el desarrollo de las 
poblaciones (de Junio a Octubre en el caso más favorable). Sus características físicoquímicas y la composición del fitoplancton han sido estudiadas con detalle por MARTINEZ $(1975,1977,1980)$.

La comunidad zooplanctónica, relativamente diversificada, está compuesta por 6 especies de crustáceos, 6 especies de rotiferos y 2 especies de protozoos. Los copépodos constituyen el grupo más importante, siendo Mixodiaptomus laciniatus la especie dominante. Esta población se comporta en su desarrollo como acíclica (no presenta cambios en la forma de reproducción) y univoltina (una sola generación por año). La eclosión de los huevos comienza durante el deshielo y la mayoría de los individuos alcanzan el estado adulto antes de que se produzca una nueva helada. Una menor proporción de individuos, no obstante, parece soportar las condiciones desfavorables (cobertura de hielo) en forma de copepoditos en los últimos estadios de desarrollo (CIII a $\mathrm{CV}$ ), dando lugar a los adultos de gran tamaño que se observan en los primeros muestreos del siguiente ciclo (CRUZ-PIZARRO, 1981).

Estas condiciones de marcada estacionalidad del ciclo de vida y la posibilidad de seguir el desarrollo de los estadios larvarios, son idóneas para el estudio de algunas actividades metabólicas de los organismos vivos y en este contexto se aborda el presente trabajo sobre la biomasa y producción de $M$. laciniatus.

\section{MATERIAL Y METODOS}

\section{TOMA DE MUESTRAS. RECUENTO DE ORGANISMOS}

El material empleado procede una serie de muestreos periódicos realizados hacia el mediodía en la zona de máxima profundidad del lago durante el periodo libre de hielo del año 1977. Las muestras se tomaron a las siguientes profundidades: $0,1,2,4,6,8 \mathrm{y}$, cuando fue posible, 10 y $11 \mathrm{~m}$. Los días de muestreo están especificados en la tabla III.
En la toma de muestras se utilizó una botella de Van Dorn doble con 81 . recogidos a cada profundidad que se filtraron a través de un disco de red de nytal de $40 \mu \mathrm{m}$. de diámetro de poro el cual se conserva en formol al 4\%. En cada uno de los días de muestreo se realizó, además, un arrastre vertical, desde el fondo a la superficie, con una red simple de plancton de un metro de longitud, $30 \mathrm{~cm}$. de diámetro de boca y malla y de $40 \mu \mathrm{m}$. El material recogido en un colector de vidrio, se conservaba como en el caso anterior.

El recuento de los organismos se hizo previa sedimentación durante 24 horas en cámaras tipo Utermohl de $50 \mathrm{cc}$. de capacidad de las muestras cuantitativas después de lavar el filltro. Posteriormente se contaron, mediante un microscopio invertido, todos los individuos presentes en la placa basal de la cámara con distinción de cada uno de los estadios larvarios de nauplio (N1 a N6) y copepodito (CI a $\mathrm{CV}$ ) y ambos sexos entre los adultos.

\section{MEDIDAS EFECTUADAS}

Los datos sobre la longitud de los diferentes estadios, se obtuvieron de la medida directa (mediante micrómetro ocular) de 2.302 individuos procedentes de arrastres verticales. Los valores de densidad están expresados como org $/ \mathrm{m}^{3}$ y se obtuvieron al dividir los valores resultantes (en org $/ \mathrm{m}^{2}$ ) de integrar los datos procedentes de las diferentes profundidades muestreadas, por la profundidad máxima del lago, en cada caso.

\section{RESULTADOS}

\section{CURVA DE MORTALIDAD}

La figura 1, muestra la densidad de organismos presentes en cada estadio y da una idea de la evolución de la población durante el período de estudio. Se observa que el dia 28 de Julio, cuando el lago presenta aún una gran cantidad de hielo, la población está compuesta únicamente por nauplios en el 


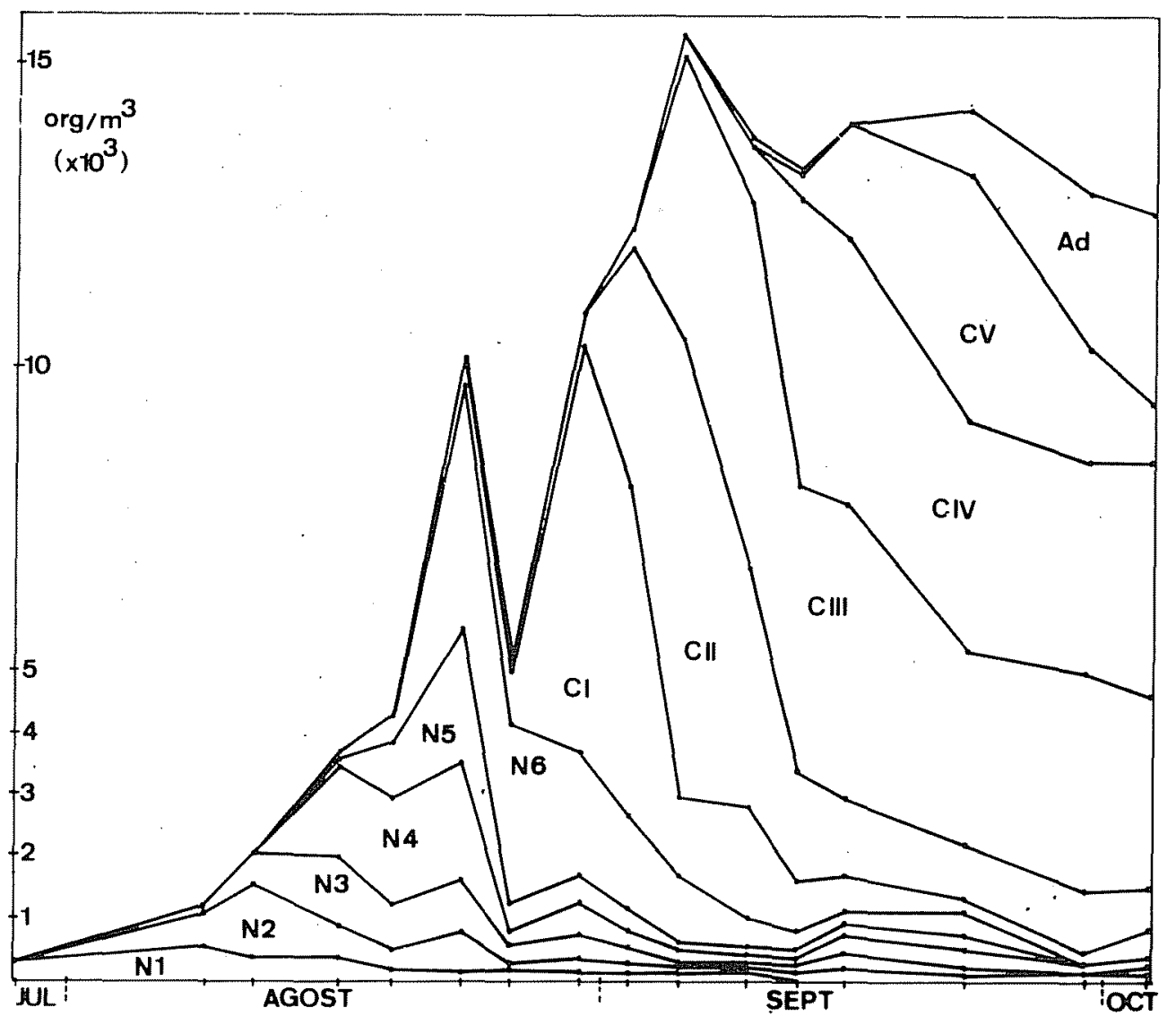

Fig. 1. Densidad y composición en estadios nauplios (N1 a N6) y copepodíticos (CI a CV) de la población de $M$. laciniatus durante el período de muestreo.

primer estadio de desarrollo (N1), lo que significa que los huevos habrian empezado a eclosionar muy poco antes. Aunque a lo largo del período de muestreo se han encontrado, fundamentalmente en muestras de arrastre vertical, adultos de gran tamaño, las primeras hembras provenientes de los huevos que comienzan a eclosionar con la fusión del hielo, aparecen en el muestreo correspondiente al 22 de Septiembre.

El máximo poblacional corresponde al muestreo del 5 de Septiembre. Asumiendo que en este momento termina el periodo de eclosión de los huevos (de hecho, los últimos NI observados corresponden al muestreo del dia 9) y obviando fenómenos de migración, la disminución en el número de individuos de la población hemos de asociarla a un proceso de mortalidad, hasta entonces enmascarada por los nuevos aportes de individuos. $\mathrm{Si}$ consideramos esta mortalidad constante para todo el periodo (t), podemos suponer que las muertes son proporcionales al número de individuos presentes $(\mathrm{N})$ y a una tasa instantánea de mortalidad $(\mathrm{m})$ :

$$
\frac{d N}{d t}=-m N
$$

La ecuación que describe este decreci- 
miento de la población a partir del máximo poblacional es:

$$
N_{t}=14554.6 e^{-0.00528 t}
$$

Como la hemos calculado con referencia a dias, la tasa diaria de mortalidad (m) es, pues, del $0.528 \%$ de la población, un valor muy semejante al que REY \& CAPBLANCQ (1975) encuentran para esta especie en el lago de Port-Bielh.

\section{DURACION DEL DESARROLLO}

A partir de los datos que se muestran en la figura 1 es posible estimar la duración del desarrollo de la población entre 52 y 56 días.

La duración de cada estadio puede ser obtenida a partir de los datos del muestreo, por dos métodos distintos:

a) Considerando el tiempo transcurrido entre las primeras apariciones de individuos correspondientes a dos estadios sucesivos.

b) Midiendo la distancia, en días, entre los valores observados de máxima densidad de dos estadios sucesivos ("pulsos" medianos; COMITA, 1956; ROFF \& CARTER, 1972).

La precisión de estos métodos es función, principalmente, de la frecuencia de muestreo y tampoco es infrecuente la existencia de varios "pulsos" máximos para cada estadio, lo que introduce un error en el cálculo. Los datos del tiempo transcurrido entre dos estadios obtenidos a partir de cultivos de laboratorio (WUTHRICH, 1948; RAVERA, 1953) sólo se pueden aplicar como orientativos a un ecosistema determinado pues factores como la temperatura, luminosidad, nutrición, sexo, etc. actúan sobre la duración de cada estadio, (ECKSTEIN, 1964; AUVRAY \& DUSSART, 1966).

Por todo ello, hemos preferido utilizar el método de cálculo empleado por REY \& CAPBLANCQ (1975) para esta misma especie y que se basa en que si $n_{1}$ es el número de individuos del estadio i presentes en el lago en el momento $t$ y $\bar{D}_{i}$ la duración media de este estadio; el número de indivi-

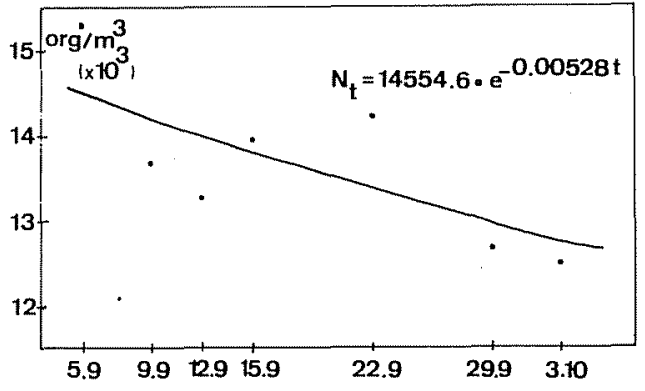

Fig. 2. Decrecimiento en tamaño de la población de M. laciniatus.

duos que pasarán al estadio siguiente en un día será:

$$
\frac{\mathrm{n}_{\mathrm{i}}}{\overline{\mathrm{D}_{\mathrm{i}}}}
$$

y durante los $\mathrm{T}$ días que dure la presencia del estadio en el medio, pasarán:

$$
\int_{0}^{T} \frac{n_{i}}{\bar{D}_{i}}
$$

valor que ha de ser igual al número medio de individuos presentes en el lago durante ese periodo $\mathrm{T}$, por tanto:

$$
\bar{D}_{i}=\frac{1}{N} \int_{0}^{T} n_{i}
$$

El valor medio de $\mathrm{N}$ para el periodo considerado se ha estimado utilizando la curva de mortalidad de la figura 2.

Los resultados obtenidos se presentan en la tabla I. La duración media de los tres últimos estadios copepodíticos (CIII, CIV y CV) es muy difícil de precisar pues no se conoce el tiempo de permanencia de cada uno de ellos en el medio ( $\mathrm{T}$ ) ya que continúan estando presentes después del último de los muestreos realizados e incluso, con elevados valores de densidad (CIII y CIV). Con el método de cálculo utilizado, sólo podemos saber que la duración media de estos estadios debe ser, respectivamente, mayor de $9.14,6.84$ y 3.87 días. 
Para los cálculos posteriores de Producción, hemos estimado los valores de D de CIII y CIV como los valores enteros (en días) superiores y más próximos a los obtenidos con el método general de cálculo. En el caso de CV, la estimación se ha hecho como la diferencia entre la duración del desarrollo de la población y la suma de las duraciones medias de todos los estadios anteriores.

\section{CALCULO DEL PESO DE LOS INDIVIDUOS}

La relación que existe entre la longitud de un individuo de un estadio de desarrollo determinado y su peso, está ampliamente demostrado y muchos autores la han tratado de cuantificar, bien a partir de medidas directas de ambas magnitudes en los diferentes estadios o bien asociando los individuos a formas geométricas simples. Se han propuesto así una serie de expresiones que, con un cierto grado de imprecisión, permiten una aproximación al peso de un individuo a partir de su longitud, al menos en los estadios de copepodito y adulto.

En nuestro caso, hemos utilizado la expresión que establecen REY \& CAPBLANCQ (1975) para M. laciniatus del lago de PortBielh a partir de los pesos de lotes de individuos agrupados por estadios:

$$
\mathrm{W}_{\mathrm{mg}}=0,00714 \mathrm{~L}_{\mathrm{mm}}^{3,8}
$$

La tabla II muestra los pesos medios correspondientes a cada uno de los estadios $\left(\mathrm{p}_{\mathrm{i}}\right)$ en el lago de La Caldera utilizando esta expresión. Como $L$, en cada caso, se ha tomado el valor medio de los medianos obtenidos en cada día de muestreo (CRUZPIZARRO, 1981).

\section{BIOMASA TOTAL}

El cálculo de la biomasa en cada uno de los muestreos está basado en la ecuación general:

$$
\mathrm{B}=\mathrm{n}_{0} \mathrm{p}_{0}+\mathrm{n}_{1} \mathrm{p}_{1}+\mathrm{n}_{2} \mathrm{p}_{2}+\ldots .+\mathrm{n}_{\mathrm{i}} \mathrm{p}_{\mathrm{i}}
$$

donde $n_{0}, n_{1}, n_{2} \ldots n_{i}$ es el número de individuos presentes de cada estadio y $\mathrm{p}_{0}, \mathrm{p}_{1}$, $\mathrm{p}_{2} \quad \ldots \mathrm{p}_{\mathrm{i}}$ es el peso medio de un individuo de cada estadio de desarrollo, desde huevo hasta adulto. Los valores obtenidos de este cálculo se muestran en la tabla III.

\section{CRECIMIENTO PONDERAL DE LOS INDIVIDUOS}

El peso medio de un individuo de la población $(\overline{\mathrm{p}})$ en cada muestreo se estimó como la relación entre la biomasa y el número total de individuos $(\mathrm{N})$ en el mismo:

$$
\overline{\mathrm{p}}=\frac{\mathrm{B}}{\mathrm{N}}
$$

Los valores obtenidos para cada día, se presentan en la tabla III y la figura 3 (linea continua) muestra su evolución a lo largo del periodo de estudio.

El aumento diario del peso medio de un individuo de la población $(\Delta \overline{\mathrm{p}})$ entre dos muestreos sucesivos $\left(t_{1}\right.$ y $\left.t_{2}\right)$ se calculó como:

$$
\Delta \bar{p}=\frac{\bar{p}_{2}-\bar{p}_{1}}{t_{2}-t_{1}}
$$

TABLA I. Duración media (en días) de los diferentes estadios larvarios.

Estadio

dias

NI

N2

N3 1,32

N4 1,91

N5 1,46

N6 3,58

CI 4,60

CII 4,50

CIII 10,00

CIV $\quad 7,00$

CV 15,00 
TABLA II. Valores de longitud, peso y aumento diario del peso medio de cada estadio.

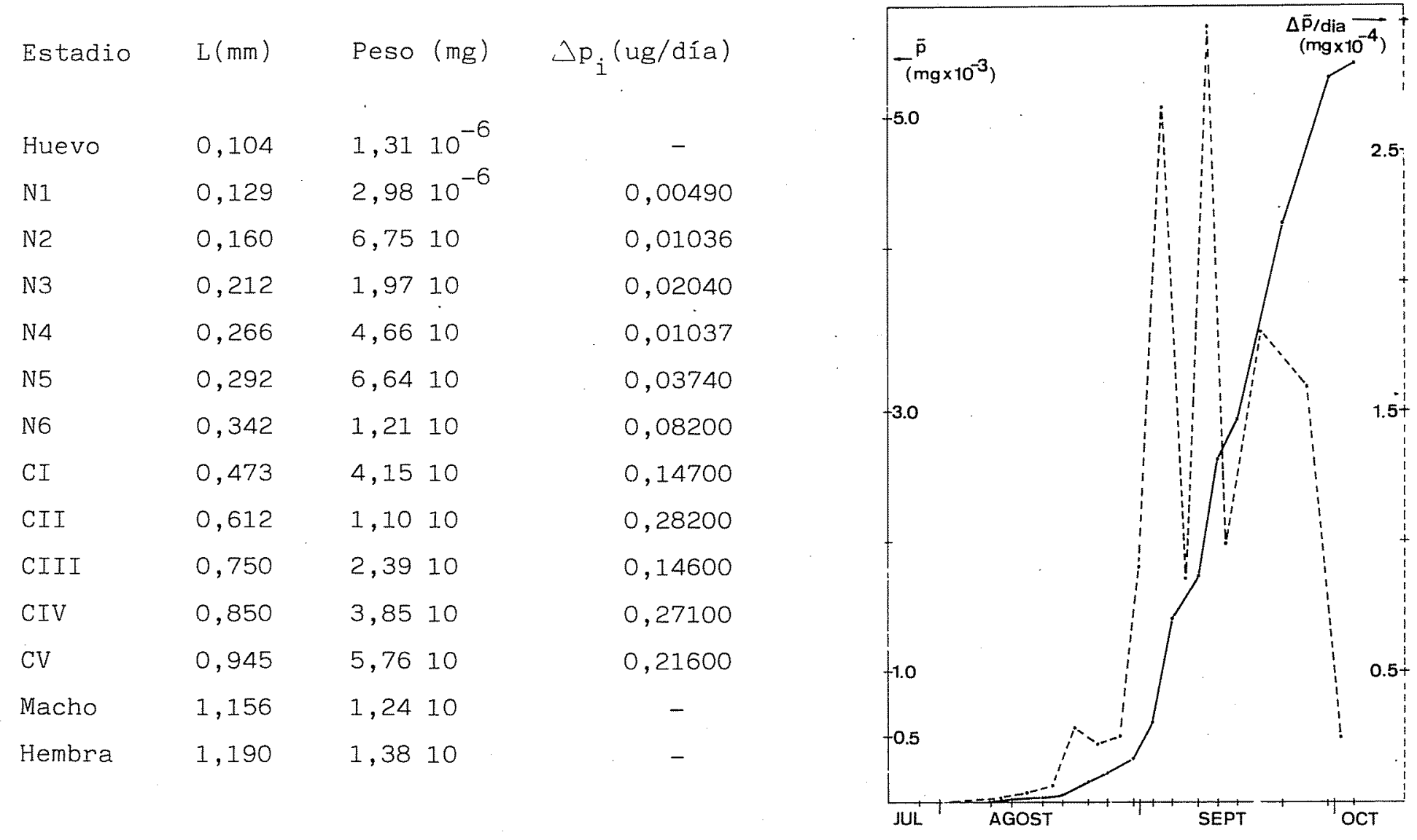

Fig. 3. Evolución del peso medio de un individuo de la población (ā) (línea continua) y de su incremento diario $(\Delta \bar{p} /$ día) (línea de trazos). 
TABLA III. Valores de biomasa, producción y del cociente producción/biomasa $(\mathrm{P} / \mathrm{B})$ durante el período de estudio del año 1977.

\begin{tabular}{|c|c|c|c|c|c|c|c|}
\hline $\begin{array}{l}\text { Fecha } \\
\text { muestreo } \\
\text { (1977) }\end{array}$ & $\begin{array}{c}N \\
\left(\mathrm{org} / \mathrm{m}^{3}\right)\end{array}$ & $\begin{array}{l}\text { Peso medio } \\
\bar{p} \text { (mg) }\end{array}$ & $\begin{array}{l}\hat{厶} \overline{\mathrm{p}} / \mathrm{dia} \\
\quad(\mathrm{mg})\end{array}$ & $\begin{array}{c}B \\
\left(\mathrm{mg} / \mathrm{m}^{3}\right)\end{array}$ & $\begin{array}{c}P \\
\left(\mathrm{mg} / \mathrm{m}^{3} \text { dia }\right)\end{array}$ & $\begin{array}{c}\mathrm{P}^{\prime} \\
\left(\mathrm{mg} / \mathrm{m}^{3} \mathrm{dia}\right)\end{array}$ & $\mathrm{P}^{\prime} / \mathrm{B}$ \\
\hline 28-VII & 273 & $2,9810^{-6}$ & & $8,1310^{-4}$ & & 0,0013 & 1,60 \\
\hline 8-VIII & 1062 & $5,7310^{-6}$ & $2,5010^{-7}$ & $6,0910^{-3}$ & $\begin{array}{l}1,6710^{-4} \\
1,8610^{-3}\end{array}$ & 0,0089 & 1,46 \\
\hline II-VIII & 2059 & $9,3110^{-6}$ & $4,1610^{-6}$ & 0,0192 & 1,8610 & 0,0251 & 1,31 \\
\hline 16-VIII & 3659 & $3,0110^{-5}$ & $5,7010^{-6}$ & 0,1101 & 0,0226 & 0,0532 & 0,48 \\
\hline 19-VIII & 4279 & $4,7210^{-5}$ & $2,8710^{-5}$ & 0,2019 & 0,2076 & 0,0997 & 0,49 \\
\hline 23-VIII & 10191 & $1,6210^{-4}$ & $2,2710^{-5}$ & 1,6482 & 0,1729 & 0,4910 & 0,33 \\
\hline $2 \theta-V I I I$ & 5045 & $2,3010^{-4}$ & $2,6510^{-5}$ & 1,1613 & 0,2111 & 0,3532 & 0,30 \\
\hline $30-V I I I$ & 10886 & $3,3610^{-4}$ & $8,8010^{-5}$ & 3,6571 & 1,0170 & 1,3158 & 0,36 \\
\hline $2-X I X$ & 1228 & $6,0010^{-4}$ & $2,6710^{-4}$ & 7,3433 & 3,6989 & 2,1127 & 0,29 \\
\hline $5-X I X$ & 15473 & $1,4010^{-3}$ & $8,5010^{-5}$ & 21,7258 & 1,2422 & $3 ; 1811$ & 0,15 \\
\hline $9-X I X$ & 13747 & $1,7410^{-3}$ & $2,9710^{-4}$ & 23,8927 & 4,0148 & 2,5719 & 0,11 \\
\hline $12-X I X$ & 13287 & $2,6310^{-3}$ & $9,6710^{-5}$ & 34,8967 & 13191 & 2,7278 & 0,08 \\
\hline $15-X I X$ & 13996 & $2,9210^{-3}$ & $1,8310^{-4}$ & 40,8354 & 2,5854 & 2,7860 & 0,07 \\
\hline $22-X I X$ & 14260 & $4,2010^{-3}$ & $1,5710^{-4}$ & 59,8615 & 2,1212 & 2,7219 & 0,04 \\
\hline 29-XIX & 12529 & $\begin{array}{l}5,3010^{-3} \\
5,4010^{-3}\end{array}$ & $2,5010^{-5}$ & 67,6409 & 0,3161 & 2,0076 & 0,03 \\
\hline
\end{tabular}


siendo $\overline{\mathrm{p}}_{1}$ y $\overline{\mathrm{p}}_{2}$ los pesos medios de un individuo de la población en ambos muestreos. Los valores de $\Delta \bar{p} /$ día se muestran en la tabla III y en la figura 3 (linea de trazos).

\section{CALCULO DE LA PRODUCCION}

La Producción (P) entre dos muestreos sucesivos se ha evaluado a partir del incremento diario del peso medio de un individuo $(\Delta \overline{\mathrm{p}} / \mathrm{d}$ ía) y del número medio de individuos $\left(\mathrm{N}_{1}+\mathrm{N}_{2} / 2\right)$ durante ese período. Por tanto:

$$
P_{1-2}=\left(\frac{\bar{p}_{2}-\bar{p}_{1}}{t_{2}-t_{1}}\right) \times\left(\frac{N_{1}+N_{2}}{2}\right)
$$

Como método comparativo hemos estimado la Producción en cada muestreo ( $\left.\mathrm{P}^{\prime}\right)$ basándonos en el crecimiento diario medio (en peso) de los diferentes estadios $\left(\Delta \mathrm{p}_{\mathrm{i}} / \mathrm{d}\right.$ ía) $y$ en el número de individuos de cada estadio $\left(n_{\mathfrak{i}}\right)$. Entonces:

siendo

$$
\mathrm{P}^{\prime}=\sum_{\text {huevo }}^{\text {Ad }} \Delta \overline{\mathrm{p}}_{\mathbf{i}} \cdot \mathrm{n}_{\mathbf{i}}
$$

$$
\Delta \overline{\mathrm{p}}_{\mathrm{i}}=\frac{\overline{\mathrm{p}}_{\text {final }}-\overline{\mathrm{p}}_{\text {inicial }}}{\overline{\mathrm{D}}_{\mathrm{i}}}
$$

y $\bar{D}_{i}$, la duración media de cada estadio. Se considera como $\overrightarrow{\mathrm{p}}_{\text {final }}$ de un estadio, el peso medio del siguiente. En la tabla II se dan los valores de $\Delta \overline{\mathrm{p}}_{\mathbf{i}}$ para cada estadio de $\mu \mathrm{g} / \mathrm{dia}$ y su representación gráfica se muestra en la figura 4 (línea de trazos). Los valores de $\mathrm{P}$ y $P^{\prime}$ se muestran en la tabla III.

\section{DISCUSION}

\section{DURACION DE LOS ESTADIOS}

De los resultados que se presentan en la tabla I se observa que la duración de cada estadio suele ser (particularmente para los primeros estadios), menor que la calculada para esta especie en ecosistemas semejantes (REY \& CAPBLANCQ, 1975) e incluso que la obtenida en experiencias de laboratorio.

El ciclo biológico particular de esta especie en el lago de La Caldera, en el que una gran parte de la población alcanza el estadio adulto al final de la época de deshielo, mientras otra parte de ella parece sobrevivir al periodo de helada en forma de CIII y CIV principalmente (CRUZ-PIZARRO, 1981), deja entrever la posibilidad de una bimodalidad en cuanto a la duración media de los estadios. No es aventurado pensar que los primeros individuos que alcanzan los estadios CIII y CIV, tengan una D mucho menor que los que llegan a tales estadios más retrasados en el tiempo, cerca del comienzo de la época de heladas, que parecen tener un "ralentizamiento" en su desarrollo (atribuible fundamentalmente a un descenso de la temperatura) y permanecen mucho más tiempo en el mismo estadio.

\section{BIOMASA. PRODUCCION. RELACION P/B}

Los valores de Biomasa, comprendidos entre $8.1310^{-4}$ y $67,6409 \mathrm{mg} / \mathrm{m}^{3}$, siguen a lo largo del periodo de estudio (fig. 5) una variación paralela a la del peso medio de un individuo de la población ( $\bar{p})$. Esto era de esperar en función de la variación observada en el peso medio de cada estadio $\left(\dot{\bar{p}}_{\mathfrak{i}}\right)$ en este mismo período.

El incremento tan notable de peso en el paso de CIV a CV, junto con la menor duración media de CIV en relación con CIII y CV, determina la existencia de los dos máximos observados tanto en el $\Delta \overline{p_{i}} /$ dia (fig. 4 ; linea de trazos), como en $\Delta \overline{\mathrm{p}} /$ día (fig. 3 , línea de trazos), cuya evolución paralela, por otro lado, se acentúa por la semejanza entre la frecuencia de los muestreos (en días) y la duración media de los estadios.

Aunque el incremento de Biomasa es continuo durante el periodo de existencia de la población, es posible diferenciar dos etapas en cuanto a su tasa diaria de aumento $\left(\mathrm{P}^{\prime} / \mathrm{B}\right)$. Un primer periodo en el que la razón $\mathrm{P}^{\prime} / \mathrm{B}$ presenta valores superiores a 0.3 , que corresponde a la fase de crecimiento en número de individuos y en la que el aumento de Biomasa es paralelo a la variación del peso 


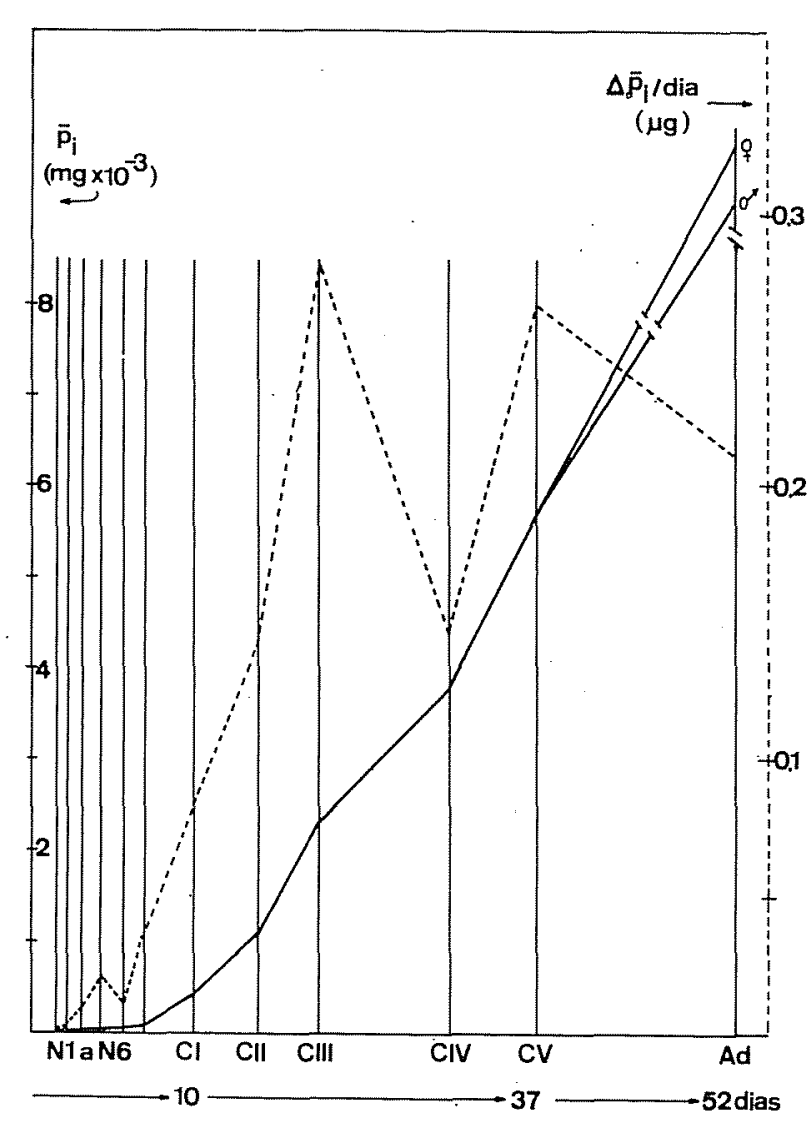

$\longrightarrow$ Fig. 4. Evolución del peso medio de cada estadio $\left(\overline{\mathrm{p}}_{\mathfrak{i}}\right)$ (línea continua) y de su incremento diario $\left(\Delta \bar{p}_{\mathrm{j}} / \mathrm{d}\right.$ ía) (linea de trazos).

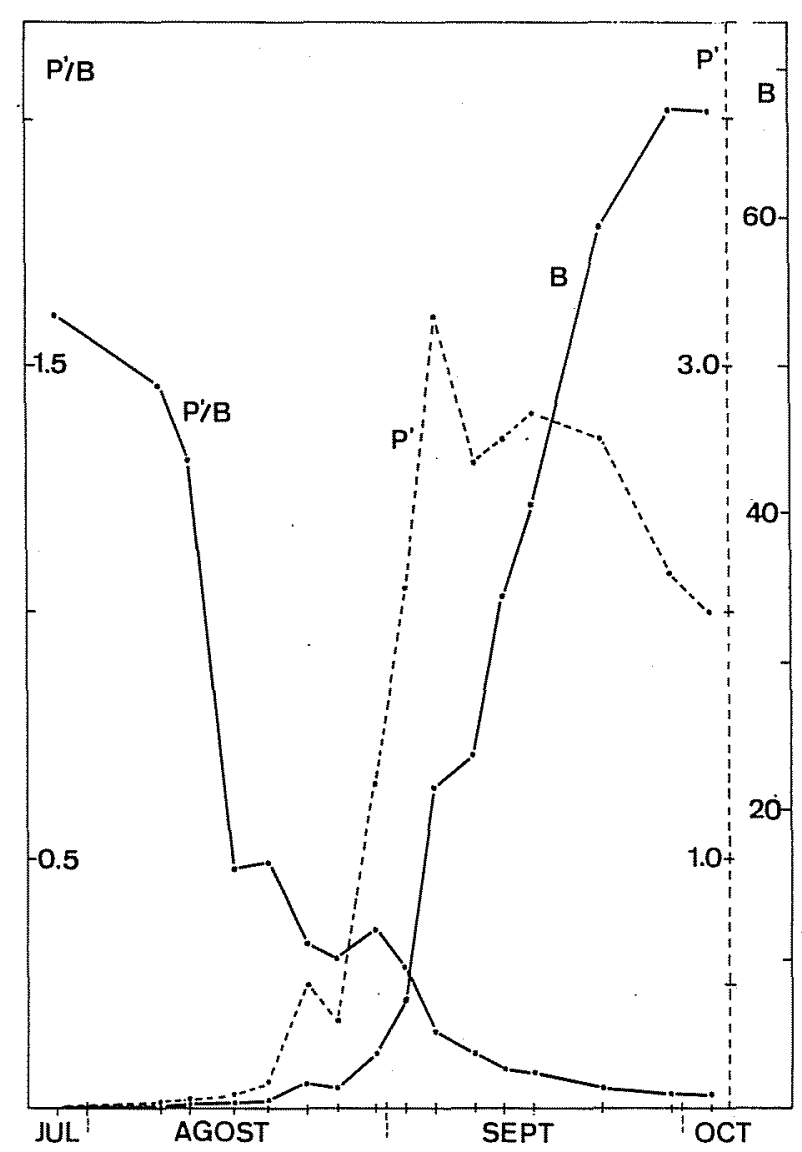

Fig. 5. Biomasa $\left(\mathrm{mg} / \mathrm{m}^{3}\right)$, Producción ( $\mathrm{mg} / \mathrm{m}^{3}$. día) y relación Producción/Biomasa (P/B) durante el período de estudio. 
medio de cada estadio y una segunda etapa en la que dicho cociente disminuye hasta alcanzar el valor de 0.03 al final del periodo libre de hielo y que corresponde al decrecimiento en individuos de la población, coincidiendo en la época en que los individuos están llegando en su desarrollo a los últimos estadios copepodíticos y de adulto.

La Producción empieza a ser importante desde mediados de Agosto y alcanza su máximo en el muestreo correspondiente al dia 5.9.77, manteniéndose en niveles elevados durante la mayor parte del mes de Septiembre para experimentar un descenso considerable al final de este mes.

Las mayores diferencias en los valores de Producción obtenidos por los métodos empleados dos (P y P') se producen durante los muestreos correspondientes a los días
29.9 y 3.10 .77 (tabla III). Esto es lógico si pensamos que la Biomasa en los dos últimos muestreos es casi idéntica, lo mismo que ocurre con el número de individuos, de manera que cuando para el cálculo de $\mathrm{P}$ usamos las diferencias diarias del peso medio de los individuos $(\Delta \overline{\mathrm{p}} / \mathrm{dí})$, obtenemos un valor sustancialmente menor que si comparamos los incrementos diarios en peso de cada estadio $\left(\Delta \bar{p}_{\mathrm{i}} /\right.$ día $)$ y el número medio de individuos en cada uno de ellos $\left(\mathrm{n}_{\mathrm{i}}\right)$.

Los valores de Biomasa, Producción asi como del incremento diario de la Biomasa obtenidos son, en general, comparables a los calculados para esta especie en ecosistemas acuáticos de montaña semejantes (REY \& CAPBLANCQ, 1975) e incluso que los que se citan para larvas de insectos en estos ambientes (GIANI \& LAVILLE, 1973).

\section{BIBLIOGRAFIA}

AUVRAY, C. \& DUSSART, B. 1966 . Role de quelques facteurs du milieu sur le développement postembryonaire des Cyclopides (CrustacésCopépodes). I. Génćralites. Cas de Eucyclops. Bull. Soc. Zool. Fr., 91:477.491.

COMITA, G.W. 1956. A study of a Calanoid Copepod population in an arctic lake. Ecology. $37(3): 576-591$

CRUZ PIZARRO, L. 1981. Estudio de la comunidad zooplanctónica de un lago de alta montaña (I. Caldera, Sierra Nevada, Granada). Tesis Doctoral. Universidad de Granada, 180 págs.

I:CKSTEIN, H. 1964. Untersuchungen über den lïnfluss des Rheinwassers auf die Limnologie des Schluchsees. Arch. Hydrobiol., 28(5): 119182.

CiANI, N. \& LAVILLE, H. 1973. Cycle biologique et Production de Sialis lutaria L. (Megaloptera) dans le lac de Port-Bielh (Pyrénées Centrales). Annls. Limnol., 9(1) 45-61.

MARTINLZ, R. 1975. Firsts report on the limnology of the alpine lake La Caldera, in the Penibetic Mountains (Sicrra Nevada, Granada, Spain). Verh. Intemat. Verein. Limnol, 19 : 1.133-1.139.
MARTINEZ, R. 1977. Phy toplankton species, biomans and diversity in lake La Caldera (Sierra Nevada, Granada, Spain) Acta Hydrobiol., 19 (2): 95-107.

1980. Seasonal variation of phytoplankton biomass and photosynthesis in the high-mountain lake La Caldera (Sierra Nevada, Spain). In: De velopments in Hydrobiology (DOKULIL et al eds.): 111-119. The Hague.

RAVI:RA, O. 1953. Gli stadi di sviluppo dei copepodi pelagici del Lago Maggiore. Mem. Ist. Ital. Idrobiol. 7: 129-151.

REY, J. \& CAPBLANCQ, J. 1975. Dynamique des populations et production du zooplankton de lac de Port-Bielh (Pyrénées Centrales). Annls. Limnol., 11:1-45.

ROFF, J.C. \& CARTER, J.C.H. 1972. Life cycle and seasonal abundance of copepod Limnocala nus macrurus SARS in a high arctic lake. Limnol. Occanogr., 17(3): 363-370.

WUTHRICH, M. 1948. Etude du développement des nauplii de Diaptomus gracilis SARS et Diaptomus laciniatus LILLJEBORG. Rev. Suisse Zool., 55: 427-445. 